\title{
Spectroscopy of Highly Excited States of the Hydrogen Atom
}

\author{
Simon Scheidegger ${ }^{\S *}$ and Frédéric Merkt* \\ §SCS-DSM Award for best poster presentation in Physical Chemistry
}

\begin{abstract}
In this contribution, we describe the status of the development of a precision-spectroscopic experiment aimed at measuring transitions to states of high principal quantum number $n$ of the hydrogen atom $(\mathrm{H})$. These states form series (called Rydberg series) which converge for $n \rightarrow \infty$ to the ionization threshold of $H$. The ionization energy of $\mathrm{H}$ can thus be determined directly by measuring the frequencies of transitions to high- $n$ states and extrapolating the Rydberg series to $n \rightarrow \infty$.
\end{abstract}

Keywords: High resolution $\cdot$ Hydrogen atom $\cdot$ Rydberg states $\cdot$ Spectroscopy

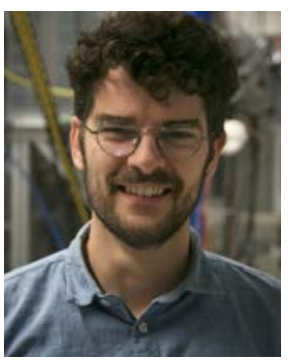

Simon Scheidegger completed his master's degree in Interdisciplinary Sciences at ETH Zurich in 2017 with focus on physics and physical chemistry. During his studies he carried out research projects in molecular physics and spectroscopy at ETH (group of Frédéric Merkt) and at the VU Amsterdam (group of Wim Ubachs). He then worked on his master thesis in precision physics at low energies (group of Klaus Kirch at ETH/ PSI) before joining the Physical Chemistry Laboratory of ETH Zurich to work on precision spectroscopy of few-electron atoms. His interests include high-precision measurements to probe fundamental properties of simple systems and the corresponding underlying theory.

\section{Introduction}

In most introductory textbooks on atomic and molecular physics and on spectroscopy, one reads that the energy levels $E_{n}$ of the hydrogen atom are described by

$$
\frac{E_{n}}{h c}=R_{\infty} \frac{\mu_{e}}{m_{e}}\left(1-\frac{1}{n^{2}}\right) .
$$

In Eqn. (1) $R_{\infty}$ is Rydberg's constant, $\mu_{e}=\frac{m_{e} m_{p}}{m_{e}+m_{p}}$ is the reduced mass of the electron-proton system, and $n$ is the principal quantum number $(n=1,2, \ldots, \infty)$. Eqn. (1) can be derived either from Bohr's atom model[1] or by solving the nonrelativistic timeindependent Schrödinger equation for the two-particle (electron, proton) system. ${ }^{[2]}$ According to Eqn. (1), the ionization energy of $\mathrm{H}(1 \mathrm{~s})$ is $R_{\infty} \frac{\mu_{e}}{m_{e}}$ and could be determined by measuring the frequency $v_{n_{1} \leftarrow n_{2}}$ of a single transition between two states of principal quantum numbers $n_{1}$ and $n_{2}$

$$
v_{n_{1} \leftarrow n_{2}}=c R_{\infty} \frac{\mu_{e}}{m_{e}}\left(\frac{1}{n_{2}}-\frac{1}{n_{1}}\right) \text {, }
$$

which, for $n_{2}=2$ and $n_{1}>2$, corresponds to Balmer's formula. ${ }^{[3]}$
The reality is more complex. When transitions of the $\mathrm{H}$ atom are measured at high resolution, deviations of the observed frequencies from those calculated by Eqn. (2) become observable, and a satisfactory description of the energy-level structure of $\mathrm{H}$ requires the solution of the relativistic two-particle wave equation (see, e.g., Barker and Glover ${ }^{[4]}$ ) and the evaluation of quantumelectrodynamics corrections. ${ }^{[5,6]}$ Even the finite size of the proton needs to be taken into account. ${ }^{[7,8]}$

Eqn. (2) with $n_{2}=2$ and $n_{1}=3,4,5$ and 6 adequately describes the spectral positions of four lines of the Fraunhofer's spectrum of the $\operatorname{sun}^{[9]}$ even when these were measured more precisely by Ångström ${ }^{[10]}$ (see Fig. 1a). Balmer's formula was used to assign further lines in the spectra of stars, an early example being the spectrum of the star Vega which contains lines with $n_{1}$ up to $15 .[11,12]$ The corresponding laboratory spectrum recorded by Herzberg ${ }^{[13]}$ also clearly reveals Balmer lines $\left(n_{2}=2\right)$ with $n_{1}$ values up to 15 , labeled $\alpha, \beta, \gamma, \ldots$ to indicate values of $n_{1}-n_{2}=$ $1,2,3, \ldots$, respectively (see Fig. 1b). Today transitions involving $n$ and $n_{2}$ values far beyond 100 are known from radioastronomy[14] (see Fig. 1c).

Precision laboratory measurements of the hydrogen-atom spectrum have played an important role in the development of the theory of atomic and molecular structure. The current understanding of the spectrum of $\mathrm{H}$ resulted from research carried out over more than 100 years, during which new and unexpected experimental results regularly challenged theory, and theoretical predictions stimulated experiments at ever increasing precision. This interplay of experiment and theory led to the discovery of fundamental phenomena.

Selected highlights are: $(i)$ the observation of the splitting of the $2 \alpha$ line by Michelson and Morley, ${ }^{[15]}$ which can be regarded as a motivation for Sommerfeld's treatment of elliptical orbits including relativistic mass scaling ${ }^{[16]}$ and Dirac's relativistic quantum theory of an electron in a central potential. ${ }^{[17]}$ The electron spin emerged naturally from Dirac's theory, resulting in corrections $\Delta E_{n, j}^{\mathrm{D}}$ of the level energies expressed in terms of half-integer total electron-angular-momentum quantum numbers $j$; (ii) the precise measurement, by Lamb and Retherford, of the $2{ }^{2} \mathrm{~S}_{1 / 2} \rightarrow 2{ }^{2} \mathrm{P}_{1 / 2,3 / 2}$ transitions, ${ }^{[18,19]}$ the frequencies of which could 


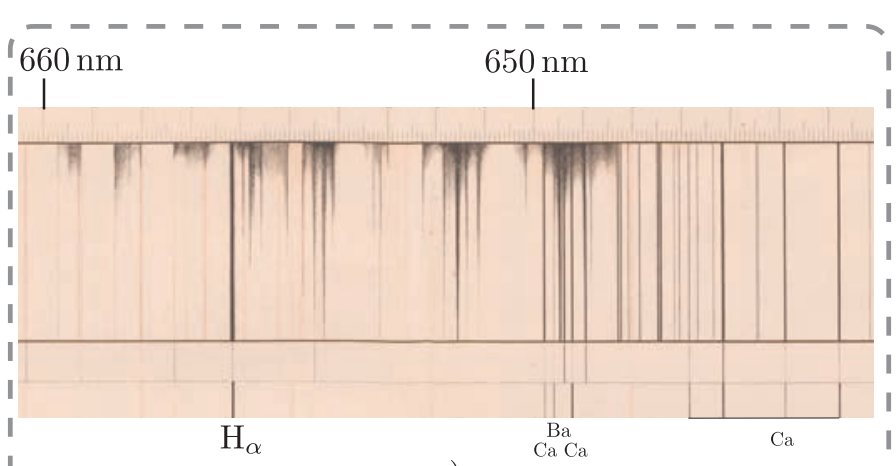

a)
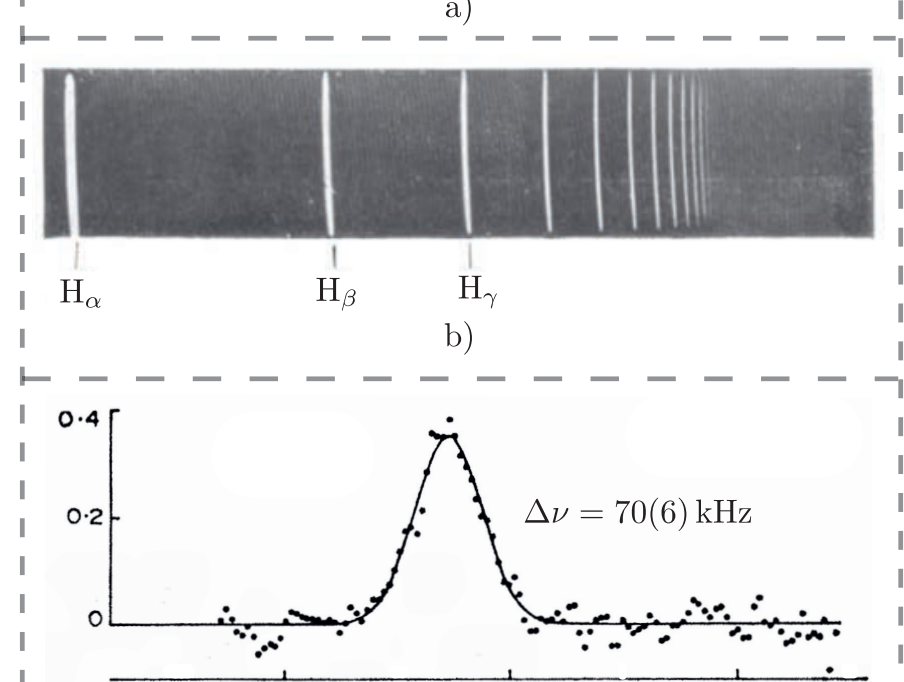

$613.200 \mathrm{MHz} \quad 613.405 \mathrm{MHz} \quad 613.610 \mathrm{MHz}$

c)

Fig. 1. a) Section of the Fraunhofer spectrum of the sun measured by Ångström (adapted from ref. [10]). The $\mathrm{H}_{\alpha}\left(n_{2}=2\right)$ line is the $3 p \rightarrow 2 \mathrm{~s}$ transition of the hydrogen atom, the other seven assigned spectral lines are from $\mathrm{Ca}$ and $\mathrm{Ba}$. b) The first 13 spectral lines of the Balmer series measured in a gas-discharge experiment by Herzberg (adapted from ref. [13]). c) Radioastronomy spectrum of the $220 \alpha$ line observed as emission from galactic $\mathrm{H}$ II regions (adapted from ref. [14]).

not be explained by Dirac's relativistic theory and led to the development of quantum electrodynamics (QED); ${ }^{[5]}$ (iii) measurements, pioneered by Rabi and coworkers, ${ }^{[20]}$ of the hyperfine structure of the $1 \mathrm{~s}{ }^{2} \mathrm{~S}_{1 / 2}$ ground state, which had been predicted theoretically by Fermi; [21] and (iv) predictions of the breakdown of Coulomb's potential for a point-like charge at the scale of the proton charge radius $r_{\mathrm{p}},{ }^{[7,22]}$ which stimulated measurements of the proton charge radius by electron scattering ${ }^{[23]}$ and spectroscopy on $\mathrm{H}$ and muonic hydrogen. ${ }^{[24,25]}$ From these measurements, the problem known as the proton-size puzzle emerged, ${ }^{[24]}$ which is currently being resolved.[26-29]

With the development of accurate frequency standards and frequency-metrology tools such as the frequency comb, measurements of transition frequencies with a fractional uncertainty of $4.2 \times 10^{-15}$ are now possible in $\mathrm{H},{ }^{[30,31]}$ which represent essential inputs for the determination of fundamental constants. ${ }^{[32]}$

The level energies of the hydrogen atom correspond to sums of contributions

$$
E_{n, l, j, f}=E_{n}+\Delta E_{n, j}^{\mathrm{D}}+\Delta E_{n, l, j}^{\mathrm{QED}}+\Delta E_{n, l}^{\mathrm{NS}}+\Delta E_{n, l, j, f}^{\mathrm{hfs}},
$$

where $l$ and $f$ are the quantum numbers associated with the electron-orbital angular momentum and the total angular momentum including nuclear spin, respectively, $\Delta E_{n, l, j}^{\mathrm{QED}}$ is the QED correction or Lamb shift, and $\Delta E_{n, l, j, f}^{\mathrm{hfs}}$ the hyperfine-structure contribution.

Precision measurements of the spectrum of $\mathrm{H}$ have so far primarily focused on transitions between levels with $n$ up to 12. ${ }^{[27-35]}$ For $n=1$ and 2, experiments and theory have resulted in the energy-level structure depicted schematically in Fig. 2, where the different contributions to the level energies are indicated (not to scale).

In our experiments, we aim at extending these measurements to transitions to higher $n$ values from the metastable $n=2{ }^{2} \mathrm{~S}_{1 / 2} f=$ 0 level. At the highest $n$ values, the fine- and hyperfine-structure splittings become negligible, because they scale as $n^{-3}$. At $n=200$, for instance, the largest splitting, the fine-structure splitting of the $n \mathrm{p}$ series, is only $10 \mathrm{kHz}$, so that the spectrum regains a structure corresponding to Balmer's formula. Extrapolation of Balmer's series to its limit and adding the precisely known interval between the $n=1$ and $n=2{ }^{2} \mathrm{~S}_{1 / 2} f=0$ levels ${ }^{[31]}$ (see Fig. 2) provide a way to determine the ionization energy of $\mathrm{H}$, i.e., of the binding energy of the $n=1{ }^{2} \mathrm{~S}_{1 / 2} f=0$ ground state, that is independent of assumed values for the energy corrections. Systematic uncertainties caused by Doppler shifts and Stark shifts induced by stray electric fields represent the main challenges of the measurements, as discussed below.

\section{Experiment}

The experimental setup is presented schematically in Fig. 3. $\mathrm{H}$ atoms in their $n=1{ }^{2} \mathrm{~S}_{1 / 2}$ ground state are generated by laser photodissociation of gaseous $\mathrm{NH}_{3}$ at $193 \mathrm{~nm}$ with an $\mathrm{ArF}$ excimer laser. The photodissociation takes place at the end of a narrow quartz capillary mounted at the orifice of a pulsed valve. ${ }^{[36]} \mathrm{NH}_{3}$ is diluted in a carrier gas $\mathrm{CG}=(\mathrm{Ne}, \mathrm{Ar})$ in a $\mathrm{CG}: \mathrm{NH}_{3}$ volume ratio of 10:1. The $\mathrm{H}$ atoms are entrained in a supersonic beam generated by expansion of the gas mixture in vacuum. ${ }^{[37]}$ After passing a skimmer, the $\mathrm{H}$ atom beam crosses the laser beams used

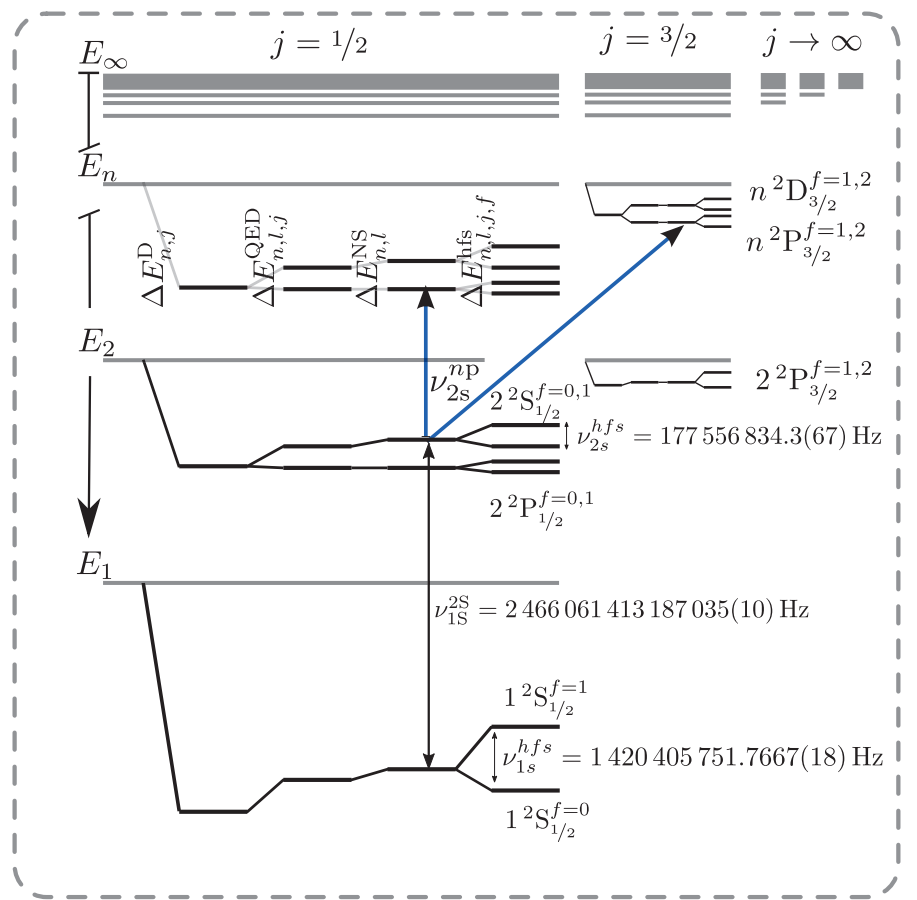

Fig. 2. Schematic representation of the energy level structure of $\mathrm{H}$ with energy corrections (not to scale) corresponding to Eqn. (3). The levels converge to the Bohr levels for $j \rightarrow \infty$ and $n \rightarrow \infty$. The arrows indicate the transitions to high $n \mathrm{p}$ Rydberg states from the metastable $2 \mathrm{~s}$ state. Selected frequencies determined in recent measurements of the hyperfine splitting in the $1 \mathrm{~s}^{[34]}$ and $2 \mathrm{~s}$ levels ${ }^{[33]}$ and of the fine- and hyperfinefree $1 \mathrm{~s}-2 \mathrm{~s}$ interval. ${ }^{[31]}$ 
for photoexcitation at right angles. The photoexcitation volume is located on the axis ( $z$ axis) of a magnetically shielded cylindrical electrode stack. Stray electric fields are compensated in three dimensions by applying potential differences across the electrode stack and across two pairs of compensation electrodes along the $x$ and $y$ axes.

The $\mathrm{H}$ atoms are first excited to the metastable $n=2{ }^{2} \mathrm{~S}_{1 / 2}$ level by nonresonant two-photon excitation using the narrow-band $(\sim 150 \mathrm{MHz}$ full width at half maximum (FWHM)), frequencytripled $(243 \mathrm{~nm})$ output of a pulsed near-Fourier-transform-limited laser operated at $729 \mathrm{~nm}$. Transitions from the metastable $n=$ $2{ }^{2} \mathrm{~S}_{1 / 2}$ state to high $n \mathrm{p}$ Rydberg states are then induced by a laser operated at $365 \mathrm{~nm}$, called $n \mathrm{p} \leftarrow 2$ s laser hereafter. The $365 \mathrm{~nm}$ radiation is produced by second-harmonic generation in a lithium triborate crystal of either a near-Fourier-transform-limited pulsed laser (bandwidth $\sim 110 \mathrm{MHz}$ ) or a single-mode continuous-wave titanium-doped-sapphire laser (cw-Ti:Sa) (bandwidth $<50 \mathrm{kHz}$ ) phase locked to a selected tooth of a frequency comb referenced to a Rb-GPS atomic clock. The transitions are detected by ionizing

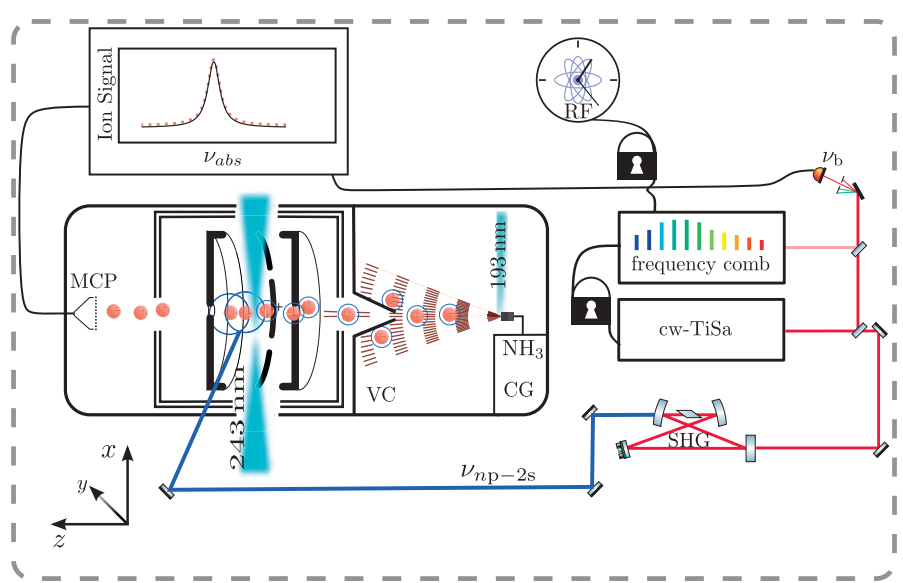

Fig. 3. Schematic view of the experimental setup. The meaning of the abbreviations is: CG: carrier gas, RF: reference frequency, SHG: secondharmonic-generation cavity, and VC: vacuum chamber. See text for details. the $n \mathrm{p}$ Rydberg states with a pulsed electric field, which is also used to accelerate the protons towards a microchannel plate detector. The spectra are recorded by monitoring the relative proton yield as a function of the laser frequency.

\section{Results}

A typical spectrum recorded with the pulsed $n \mathrm{p} \leftarrow 2$ s laser is depicted in Fig. 4a. This spectrum reveals transitions to high- $n$ Rydberg states of $\mathrm{H}$ with $n$ in the range between 163 and 220, as indicated along the assignment bar. This spectrum illustrates our ability to observe Rydberg states of $\mathrm{H}$ beyond $n=200$ in the laboratory. The spectrum demonstrates that the stray electric fields have been compensated to below $3 \mathrm{mV} / \mathrm{cm}$, which corresponds to the Inglis-Teller field at $n=220$. The Inglis-Teller field is the field $F$ at which states of neighboring $n$ values start overlapping $\left(F=\frac{1}{3 n^{5}}\right.$ in atomic units $\left.{ }^{[38]}\right)$. The $n=220$ Rydberg state is located only $68 \mathrm{GHz}$ below the ionization limit, which can be determined by extrapolation at a precision of $200 \mathrm{MHz}$ corresponding to the linewidth of the observed transitions, limited by the pulsed nature of the laser excitation and the incomplete three-dimensional strayfield compensation.

Much narrower linewidths can be obtained by carrying the $n \mathrm{p} \leftarrow 2$ s excitation with a cw laser and compensation of the stray electric fields in three dimensions to below $1 \mathrm{mV} / \mathrm{cm}$, as illustrated for the $45 p-2 s$ transition in Fig. $4 b$. In this figure, the dots correspond to experimental data points and the full line to a Lorentzian profile with a full width at half maximum of 11.2 $\mathrm{MHz}$. Each data point in the figure corresponds to a measurement time of $1 \mathrm{~s}$. The line center can be determined at a precision of 270 $\mathrm{kHz}$, which corresponds to a relative precision $\Delta v / v=3 \times 10^{-10}$, limited by the Poisson noise of the proton counts and fluctuations in the production of the metastable $n=2{ }^{2} \mathrm{~S}_{1 / 2}$ state. These fluctuations are also responsible for the irregular intensities in Fig. $4 \mathrm{a}$.

\section{Conclusions and Outlook}

The relative precision $\Delta v / v$ demonstrated in Fig. $4 \mathrm{~b}$ is only a factor 100 larger than the precision of $4 \times 10^{-12}$ required to contribute to the solution of the proton-size puzzle. In future, the precision of the current result will be improved by longer measure-

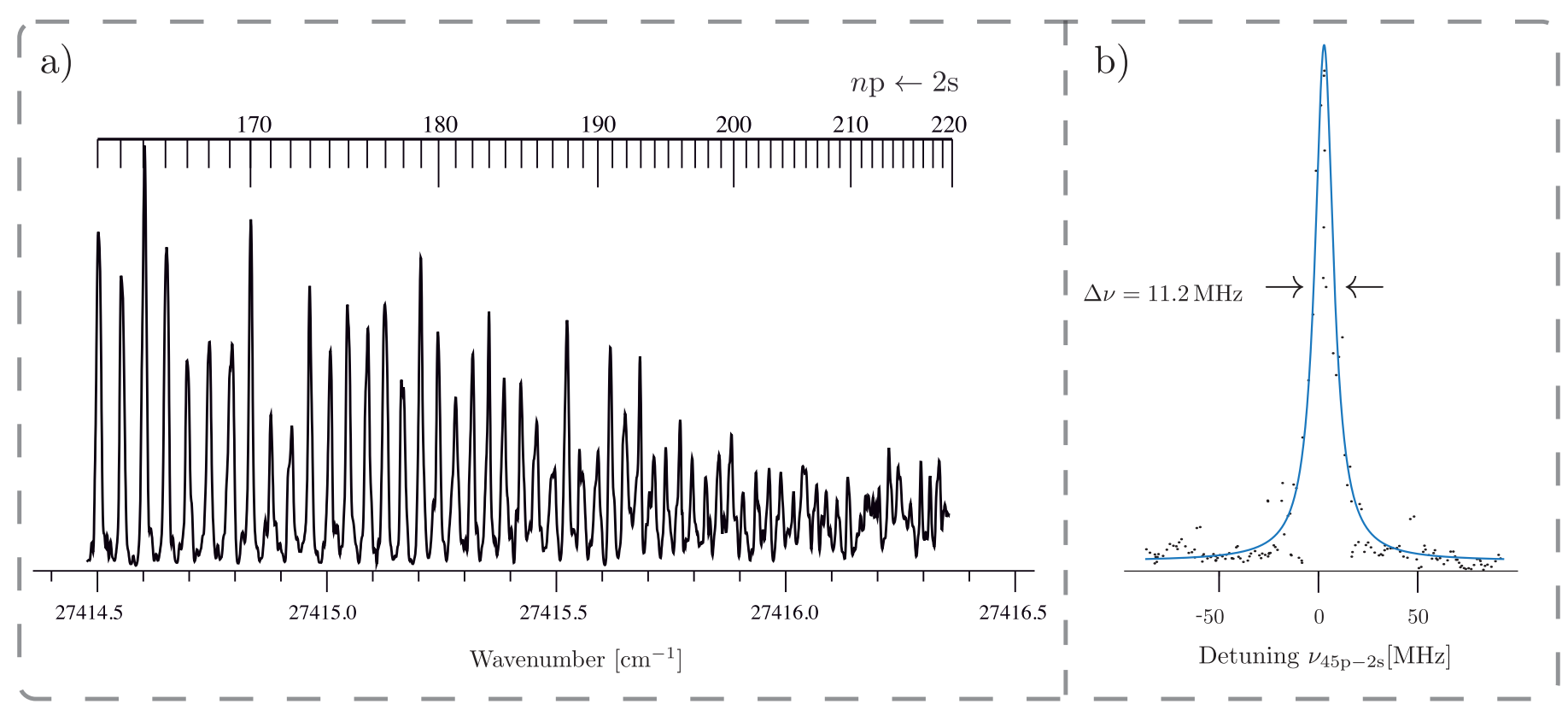

Fig. 4. a) High- $n$ spectrum of the hydrogen atom recorded from the metastable $n=2{ }^{2} S_{1 / 2}$ state with a near-Fourier-transform-limited pulsed UV laser. b) Spectrum of the $n=45$ Rydberg state of $\mathrm{H}$ recorded from the $n=2{ }^{2} \mathrm{~S}_{1 / 2}$ state using the $\mathrm{cw} n \mathrm{p}-2 \mathrm{~s}$ laser. The blue line is a Lorentzian line with a full width at half maximum of $11.2 \mathrm{MHz}$. The line center can be determined in a least-square fit (full line), with a precision limited by the experimental noise (see text for details). 
ment times, which should improve the statistical uncertainty, and a better collimation of the supersonic beam, e.g. by increasing the distance between the skimmer and the laser excitation region, which should significantly reduce the Doppler width.

The transit time of the $\mathrm{H}$ atoms in the supersonic beam $\left(\mathrm{v}_{\mathrm{H}}\right.$ $=500 \mathrm{~m} / \mathrm{s}$ ) through the $n \mathrm{p} \leftarrow 2 \mathrm{~s}$ laser beam is about $2 \mu \mathrm{s}$, which corresponds to a $\sim 500 \mathrm{kHz}$ line width and is 20 times less than the linewidth of the $45 \mathrm{p} \leftarrow 2$ s transition in Fig. $4 \mathrm{~b}$.

Reaching an absolute accuracy of $4 \times 10^{-12}$ still represents a challenge because it requires the elimination of systematic uncertainties to below $3 \mathrm{kHz}$. The two main sources of systematic uncertainty arise from the Doppler effect and from the second-order Stark effect. To obtain the Doppler-free transition frequency, the $n \mathrm{p} \leftarrow 2$ s laser needs to cross the H-atom beam in a counterpropagating, wavefront-retraced configuration with an angular deviation between the forward-propagating and reflected beams of less than $0.7 \mu \mathrm{rad}$. An uncertainty of less than $3 \mathrm{kHz}$ in the Doppler shift could be reached by verifying the overlap of the two beams using an active fiber-based retro-reflector. ${ }^{[39]}$ Reducing the stray electric fields to below $150 \mu \mathrm{V} / \mathrm{cm}$ necessitates the use of a low-noise potential source and an optimization of the fieldcompensation electrodes along the $x$ and $y$ axis. ${ }^{[40,41]}$

\section{Acknowledgments}

S. Scheidegger thanks DSM for sponsoring the best-poster-presentation award. We also thank J. A. Agner and H. Schmutz for technical support and Dr. P. Jansen for inspiring discussions and advice in the laboratory. This work is supported financially by the Swiss National Science Foundation under project No. 200020-172620 and the European Research Council (Horizon 2020, Advanced Grant No. 743121).

Received: February 28, 2020

[1] N. Bohr, London, Edinburgh, Dublin Philos. Mag. J. Sci. 1913, 26, 1.

[2] E. Schrödinger, Ann. Phys. 1926, 384, 361.

[3] J. J. Balmer, Ann. der Phys. und Chemie 1885, 261, 80

[4] W. A. Barker, F. N. Glover, Phys. Rev. 1955, 99, 317.

[5] H. A. Bethe, Phys. Rev. 1947, 72, 339.

[6] K. Pachucki, Phys. Rev. A 1995, 52, 1079.

[7] E. C. Kemble, R. D. Present, Phys. Rev. 1933, 44, 1031.

[8] G. A. Miller, Phys. Rev. C 2019, 99, 35202.

[9] J. Fraunhofer, Ann. Phys. 1817, 56, 264.

[10] A. J. Ångström, 'Recherches sur le spectre solaire: Spectre normal du soleil: atlas de six planches', Schultz, Uppsala, 1868.

[11] W. Huggins, Proc. R. Soc. London 1877, 25, 445.

[12] W. Huggins, Philos. Trans. R. Soc. London 1880, 669

[13] G. Herzberg, Ann. Phys. 1927, 389, 565.

[14] A. Pedlar, R. D. Davies, Mon. Not. R. astr. Soc. 1972, 159.

[15] A. A. Michelson, E. W. Morley, London, Edinburgh, Dublin Philos. Mag. J. Sci. 1887, 24, 463.

[16] A. Sommerfeld, Ann. Phys. 1916, 356, 1.

[17] P. A. M. Dirac, Proc. R. Scociety A 1928, 117.

[18] W. E. Lamb Jr., R. C. Retherford, Phys. Rev. 1947, 72, 241

[19] W. E. Lamb Jr, R. C. Retherford, Phys. Rev. 1950, 79, 549.

[20] J. E. Nafe, E. B. Nelson, I. I. Rabi, Phys. Rev. 1947, 71, 914.

[21] E. Fermi, Zeitschrift für Phys. 1930, 60, 320.

[22] S. Pasternack, Phys. Rev. 1938, 54, 1113.

[23] R. Hofstadter, R. W. McAllister, Phys. Rev. 1955, 98, 217.
[24] R. Pohl, A. Antognini, F. Nez, F. D. Amaro, F. Biraben, J. M. R. Cardoso, D. S. Covita, A. Dax, S. Dhawan, L. M. P. Fernandes, A. Giesen, T. Graf, T. W. Hänsch, P. Indelicato, L. Julien, C.-Y. Kao, P. Knowles, E.-O. Le Bigot, Y.-W. Liu, J. A. M. Lopes, L. Ludhova, C. M. B. Monteiro, F. Mulhauser, T. Nebel, P. Rabinowitz, J. M. F. dos Santos, L. A. Schaller, K. Schuhmann, C. Schwob, D. Taqqu, J. F. C. A. Veloso, F. Kottmann, Nature 2010, 466, 213.

[25] A. Antognini, F. Nez, K. Schuhmann, F. D. Amaro, F. Biraben, J. M. R. Cardoso, D. S. Covita, A. Dax, S. Dhawan, M. Diepold, L. M. P. Fernandes, A. Giesen, A. L. Gouvea, T. Graf, T. W. Hänsch, P. Indelicato, L. Julien, C.Y. Kao, P. Knowles, F. Kottmann, E.-O. Le Bigot, Y.-W. Liu, J. A. M. Lopes, L. Ludhova, C. M. B. Monteiro, F. Mulhauser, T. Nebel, P. Rabinowitz, J. M. F. dos Santos, L. A. Schaller, C. Schwob, D. Taqqu, J. F. C. A. Veloso, J. Vogelsang, R. Pohl, Science 2013, 339, 417.

[26] W. Xiong, A. Gasparian, H. Gao, D. Dutta, M. Khandaker, N. Liyanage, E. Pasyuk, C. Peng, X. Bai, L. Ye, K. Gnanvo, C. Gu, M. Levillain, X. Yan, D. W. Higinbotham, M. Meziane, Z. Ye, K. Adhikari, B. Aljawrneh, H. Bhatt, D. Bhetuwal, J. Brock, V. Burkert, C. Carlin, A. Deur, D. Di, J. Dunne, P. Ekanayaka, L. El-Fassi, B. Emmich, L. Gan, O. Glamazdin, M. L. Kabir, A. Karki, C. Keith, S. Kowalski, V. Lagerquist, I. Larin, T. Liu, A. Liyanage, J. Maxwell, D. Meekins, S. J. Nazeer, V. Nelyubin, H. Nguyen, R. Pedroni, C. Perdrisat, J. Pierce, V. Punjabi, M. Shabestari, A. Shahinyan, R. Silwal, S. Stepanyan, A. Subedi, V. V Tarasov, N. Ton, Y. Zhang, Z. W. Zhao, Nature 2019, 575, 147.

[27] N. Bezginov, T. Valdez, M. Horbatsch, A. Marsman, A. C. Vutha, E. A. Hessels, Science 2019, 365, 1007.

[28] A. Beyer, L. Maisenbacher, A. Matveev, R. Pohl, K. Khabarova, A. Grinin, T. Lamour, D. C. Yost, T. W. Hänsch, N. Kolachevsky, T. Udem, Science 2017, 358, 79.

[29] H. Fleurbaey, S. Galtier, S. Thomas, M. Bonnaud, L. Julien, F. Biraben, F. Nez, M. Abgrall, J. Guéna, Phys. Rev. Lett. 2018, 120, 183001.

[30] M. Zimmermann, C. Gohle, R. Holzwarth, T. Udem, T. W. Hänsch, Opt. Lett. 2004, 29, 310.

[31] C. G. Parthey, A. Matveev, J. Alnis, B. Bernhardt, A. Beyer, R. Holzwarth, A. Maistrou, R. Pohl, K. Predehl, T. Udem, T. Wilken, N. Kolachevsky, M. Abgrall, D. Rovera, C. Salomon, P. Laurent, T. W. Hänsch, Phys. Rev. Lett. 2011, 107, 203001.

[32] D. B. Newell, F. Cabiati, J. Fischer, K. Fujii, S. G. Karshenboim, H. S. Margolis, E. de Mirandés, P. J. Mohr, F. Nez, K. Pachucki, T. J. Quinn, B. N. Taylor, M. Wang, B. M. Wood, Z. Zhang, Metrologia 2018, 55, L13.

[33] N. Kolachevsky, A. Matveev, J. Alnis, C. G. Parthey, S. G. Karshenboim, T. W. Hänsch, Phys. Rev. Lett. 2009, 102, 213002.

[34] L. Essen, R. W. Donaldson, M. J. Bangham, E. G. Hope, Nature 1971, 229, 110.

[35] J.C. Garreau, M. Allegrini, L. Julien, F. Biraben, J. Phys. Fr. 1990, 51, 2293.

[36] S. Willitsch, J. M. Dyke, F. Merkt, Helv. Chim. Acta 2003, 86, 1152.

[37] E. Vliegen, F. Merkt, J. Phys. B At. Mol. Opt. Phys. 2006, 39, L241.

[38] T. F. Gallagher, 'Rydberg Atoms', Cambridge University Press, Cambridge, 1994.

[39] A. Beyer, L. Maisenbacher, A. Matveev, R. Pohl, K. Khabarova, Y. Chang, A. Grinin, T. Lamour, T. Shi, D. C. Yost, Th. Udem, T. W. Hänsch, N. Kolachevsky, Opt. Express 2016, 24, 17470.

[40] H. Saßmannshausen, F. Merkt, J. Deiglmayr, Phys. Rev. A 2013, 87, 32519.

[41] M. Beyer, N. Hölsch, J. A. Agner, J. Deiglmayr, H. Schmutz, F. Merkt, Phys. Rev. A 2018, 97, 12501.

\section{License and Terms}

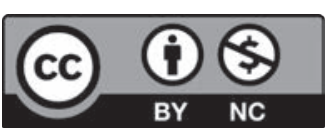

This is an Open Access article under the terms of the Creative Commons Attribution License CC BY_NC 4.0. The material may not be used for commercial purposes.

The license is subject to the CHIMIA terms and conditions: (http:// chimia.ch/component/sppagebuilder/?view=page \&id=12).

The definitive version of this article is the electronic one that can be found at doi:10.2533/chimia.2020.285 